\title{
Il romanzo digitale
}

\author{
Da Manzoni a Pirandello (e oltre)
}

\author{
Paola Italia
}

\begin{abstract}
L'articolo propone una riflessione sullo stato dell'arte del romanzo italiano nel mondo digitale svolta attraverso l'analisi dei testi in prosa della modernità presenti nel portale Biblioteca Italiana e affronta $i$ casi di studio di Manzoni, Pirandello e Tozzi, per analizzare tre progetti complementari e diversi di edizione cartacea e digitale, e di interazione tra ricerca accademica, istituzioni bibliotecarie ed editoria. I casi mostrano come il romanzo, per la sua polifonia, le sue dinamiche genetiche, la sua contaminazione con le arti visive e drammatiche, richieda una infrastruttura digitale che metta in relazione tutti questi aspetti tra loro, anche in chiave didattica, costituendo un banco di prova della necessaria trasformazione dell'edizione critica digitale in un sito di ricerca e conoscenza dei testi e dei loro autori.
\end{abstract}

This article proposes a reflection on the state of the art of the Italian novel in the digital world carried out through the analysis of modern prose texts in the website Bibliotecaltaliana.it, examining the case studies of Manzoni, Pirandello, and Tozzi, and in particular three complementary projects and several digital editions as well as interactions among academic research, institutions, librarians, and publishers. The case studies show how the novel - thanks especially to its polyphony, its genetic dynamics and contamination with the visual and dramatic arts - requires an infrastructure that also links all these aspects from an educational point of view. The novel may be considered as a testing ground for the necessary transformation of the digital critical edition into a "knowledge site" of texts and their authors.

\section{Fake Novels}

$\mathrm{N}$

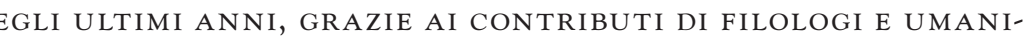
sti digitali che hanno stimolato l'incontro tra filologia e mondo digitale primo fra tutti Michelangelo Zaccarello, che ringrazio per avermi invitato a questo incontro - ho riflettuto sullo stato dell'arte dei testi della letteratura italiana in rete, per vedere cosa è cambiato non solo e non tanto nella percezione del testo, ma nell'ecdotica, nelle tecniche di edizione e nelle nuove 
modalità di presentazione e di fruizione dei testi. ${ }^{1}$ Lesperienza che ho maturato nel mondo editoriale, prima da redattore, poi da ricercatore tra case editrici e archivi editoriali, mi stimola sempre a vedere anche l'aspetto editoriale degli studi sul testo, convinta come sono che — come già auspicava Pierazzo (2015) - solo dalla collaborazione tra editoria e ricerca potranno scaturire quei modelli che hanno permesso alla rivoluzione gutenberghiana di resistere nel tempo se, ancora adesso, nonostante l'immersione digitale abbia fatto pronosticare la scomparsa del libro cartaceo, siamo ancora ben lontani dal definire quel modello un "dispositivo superato" (Bordalejo 2013 e 2016).

Vorrei oggi svolgere alcune riflessioni su un tema che non è stato ancora trattato approfonditamente e che coinvolge invece la produzione e fruizione dei testi letterari. Intendo infatti toccare la situazione del romanzo italiano nel mondo digitale, partendo dai problemi dei testi non nativi digitali, e in particolare del canone dei romanzi italiani dal Settecento al Novecento, indispensabili per capire quale sia l'infrastruttura disponibile per accoglierli e quali le modalità di fruizione, fino ad alcuni esempi tratti da testi nativi digitali, che permettono di cominciare a fare qualche considerazione sugli sviluppi della narrativa contemporanea. Ciò ci permetterà di capire se il romanzo moderno italiano richieda una specifica ecdotica digitale - come è stato sostenuto per il romanzo modernista nel mondo anglosassone - o non si tratti invece di problemi che devono essere affrontati anche per testi non romanzeschi, e non modernisti. ${ }^{2}$

Più volte, chi si è trovato a riflettere sullo statuto del testo digitale, ha lamentato l'assenza di parametri condivisi per la certificazione dell'affidabilità dei testi, e la bibliografia ora è ricca di contributi specifici in merito, da Zaccarello - che anima l'osservatorio OPEDIT sui problemi di mancato riconoscimento del riconoscimento ottico OCR - a Paolo Divizia, che ha svolto interessanti ricerche sulla tradizione di Boccaccio, mostrandoci come i testi digitali, derivati da edizioni ignote o non affidabili, tornino

1. Sul tema rimando soprattutto a Divizia 2012, Shillingsburg 2017, ZAcCaRELLO 2017a, 2017b, 2018.

2. Mi riferisco in particolare ai temi trattati inizialmente, con il caso specifico del beckettiano Watt, da Byron 2010, e recentemente dal gruppo di ricerca di Byroni Randall dell'Università di Glasgow, New modernist editing (https:// newmodernistediting.wordpress.com/), con l'esperimento svolto sul racconto di Virginia Woolf, Ode written partly in prose on seeing the name of cutbush above a butcher's shop in Pentonville https://nme-digital-ode.glasgow.ac.uk/\#), e alle riflessioni presenti nel saggio di Ross (2016): "Digital Modernism as Method: Recent Publications in Digital Humanities". 
poi nel testo cartaceo perché editori sprovveduti (a volte editori di tradizione, che dovrebbero essere pochissimo sprovveduti), preferiscono ricavare il testo dalle versioni digitali esistenti piuttosto che farlo ricomporre da una edizione cartacea affidabile.

Questo problema è imprescindibile per il romanzo digitale, che riguarda un settore più ristretto e problemi più particolari di quelli offerti dal testo digitale, ma sconta i danni del medesimo lettore, quello che qualche anno fa ho chiamato il lettore Google (It A Lia 2016). Il lettore Google è onnivoro, tende cioè a divorare tutti $\mathrm{i}$ testi presenti in rete, senza discernere tra testi buoni e meno buoni; ed è bulimico, cioè per lui conta più la quantità che la qualità: queste caratteristiche si ripercuotono sul romanzo digitale che, per analogia, si potrebbe denominare il "romanzo Google".

\section{Il Romanzo "Google"}

Il romanzo Google partecipa dello statuto del testo digitale: fluido, instabile, collaborativo e a identità autoriale collettiva. Riprenderemo questi elementi più avanti, parlando del romanzo nativo digitale. Per ora soffermiamoci sulle peculiarità del romanzo digitale non nativo, ovvero sulla presenza di romanzi della letteratura italiana in rete.

Il primo problema da affrontare è dato dallo statuto del genere. Nel portale di Biblioteca Italiana (1996-2019 [http://www.bibliotecaitaliana.it/]), l'unico che offra una banca dati amplissima di testi metadatati e certificati, che possono essere interrogati secondo diverse chiavi d'accesso, la categoria "Romanzo" non esiste:

- Commenti, traduzioni e volgarizzamenti (28)

- Documenti (167)

- Letteratura teatrale (171)

- Lettere ed epistolari (93)

- Memorialistica (50)

- Narrativa (172)

- Oratoria (38)

- Poesia (614)

- Prosa scientifica, morale e d'invenzione (6)

- Testi storici e storiografici (81)

- Traduzioni e volgarizzamenti (28)

- Trattati (468) 
Nella categoria Narrativa si trovano 172 testi che spaziano dal Duecento al Novecento (e già questo arco cronologico, di per sé, potrebbe insinuare qualche sospetto). Si tratta di una categoria molto ampia, che comprende anche testi spuri, contaminati, o testi che probabilmente non trovavano un'altra categoria in cui venire inseriti, e che non potevano costituire una categoria a sé: la Vita nova, i Reali di Francia, i Dialoghi e le Sei giornate dell'Aretino, le novelle del Bandello, Lo cunto de li cunti, I Ragguagli di Parnaso, il Decameron, la favola pastorale di Annibal Caro e finalmente - mi sono fermata alla lettera "C" - Pinocchio. Come spesso accade in rete, c'è troppo e troppo poco.

Diciamo che Lunico modo per riuscire a identificare la sezione "Romanzo" all'interno della generica sezione di "Narrativa", è ritagliare, dei 172 testi presenti nell'archivio digitale, quelli che corrispondono al genere "romanzo". Per semplificare il lavoro, ho provato a incrociare la ricerca "genere" con quella "secolo", in modo da escludere i secoli precedenti al XVIII, che - salvo alcuni tentativi fuori canone - è comunemente ritenuto la culla del grande romanzo europeo, e, come è noto, fa da incubatrice al romanzo italiano. Il sistema, però, non permette di incrociare due diverse chiavi di ricerca. Ma la mia sorpresa è stata ancora maggiore quando decisa a selezionare la sezione settecentesca eliminando i testi che non fossero corrispondenti al genere "romanzo" - mi sono accorta che mancava proprio il primo romanzo della letteratura italiana: La filosofessa italiana, o sia le avventure della Marchesa di N.N., scritte in Francese da lei medesima (Venezia, Angelo Pasinelli, 1753) di Pietro Chiari: duecentomila copie, dieci ristampe per oltre un decennio, e una seconda edizione, la Pasinelli del 1756, cui Chiari aggiunge un quarto tomo. ${ }^{3}$ Manca integralmente il Chiari, e quindi manca anche La filosofessa. A testimoniare il romanzosaggio del Settecento vi sono però le Avventure di Saffo e le Notti romane del Verri. ${ }^{4}$

Proviamo a mappare il romanzo digitale, quale ci si presenta nel portale di Biblioteca Italiana:
1. Collodi
Le avventure di Pinocchio
2. Cuoco
Platone in Italia
3. De Amicis
Cuore

3. Ripubblicato recentemente da Manni, a cura di Carlo Madrignani (2004), che propone la prima edizione del CHIARI 1753 (primi tre tomi) e del 1756 (il quarto tomo).

4. Presenti rispettivamente nell'edizione di Martinelli 1975, e di Negri 1967. 


$\begin{array}{lll}\text { 4. De Roberto } & \text { Processi verbali } \\ \text { 5. Fogazzaro } & \text { Daniele Cortis } \\ \text { 6. Fogazzaro } & \text { Piccolo Mondo Antico } \\ \text { 7. Manzoni } & \text { I Promessi Sposi } \\ \text { 8. Nievo } & \text { Le confessioni di un italiano } \\ \text { 9. Svevo } & \text { La coscienza di Zeno } \\ \text { 10. Svevo } & \text { Senilità } \\ \text { 11. Svevo } & \text { Una vita } \\ \text { 12. Tarchetti } & \text { Fosca } \\ \text { 13. Tommaseo } & \text { Fede e Bellezza } \\ \text { 14. Tozzi } & \text { Con gli occhi chiusi } \\ \text { 15. Tozzi } & \text { Il podere } \\ \text { 16. Tozzi } & \text { Tre croci } \\ \text { 17. Verga } & \text { Eros } \\ \text { 18. Verga } & \text { Eva } \\ \text { 19. Verga } & \text { I Malavoglia } \\ \text { 20. Verga } & \text { Mastro don Gesualdo } \\ \text { 21. Verri } & \text { Le avventure di Saffo } \\ \text { 22. Verri } & \text { Le notti romane }\end{array}$

Prima di vedere più in dettaglio le presenze e le assenze, colpisce il taglio cronologico che, con il 1925 della Coscienza di Zeno, ci porta a riflettere sul secondo problema legato al romanzo italiano nel mondo digitale: il copyright. È forse poco noto, e vale la pena di ricordarlo, che l'attuale normativa vigente, in vigore dal 1998, estende il diritto d'autore. Si tratta del CTEA (Copyright Term Extension Act), ovvero la norma che stabilisce per ulteriori vent'anni dagli iniziali cinquanta, stabiliti nel 1923, il divieto alla pubblicazione in assenza dell'autorizzazione degli eredi, portando la durata totale del copyright a 70 anni dopo la morte dell'autore nel caso di opere prodotte da singoli e a 120 anni dopo la data di creazione o 95 dopo l'anno di pubblicazione per le opere prodotte da un gruppo o impresa. Un'estensione imposta su pressioni della Disney, per la semplice ragione che, se fosse rimasto il limite dei cinquant'anni, la casa di produzione avrebbe perso i diritti sul suo personaggio porta bandiera: Mickey Mouse, tanto che il CTEA è noto anche come Mickey Mouse Protection Act.

Ma c'è di più. Nel trattato di pace del 1947 i paesi vincitori decisero che per i loro scrittori il periodo di 70 anni dopo la morte si sarebbe dovuto

5. Si segnala il titolo, erroneamente citato nella forma dissimilata della preposizione. 
allungare di altri 6 anni e 8 mesi, giusto il tempo di durata del secondo conflitto, durante il quale le opere non avevano avuto circolazione, e i cui diritti dovevano quindi essere tutelati per un periodo più lungo. Questa stessa possibilità non era stata data agli sconfitti. Ma nel dicembre 2006 la SIAE estende la clausola dei sei anni e otto mesi anche agli autori dei paesi sconfitti, equiparandoli ai vincitori. Le conseguenze più visibili, per la letteratura italiana del Novecento, riguardano i diritti di Pirandello (morto nel 1936), che invece di scadere nel 2006 vengono liberalizzati dal 2014 e quelli di D'Annunzio (morto due anni dopo), che lo sono dal $2016 .^{6}$

Ciò vuol dire che, per gli autori della letteratura italiana della generazione degli anni Novanta del XIX secolo, fino agli anni Venti del XX, che non siano mancati prematuramente (è il caso di Pavese, i cui diritti scadono nel 2019), solo i più giovani partecipanti a questo convegno vedranno cadere la moratoria dei diritti d'autore. In realtà, vi è stato un momento speciale, una finestra temporale tra il 2006 e il 2008 in cui si sono rese disponibili le opere di Pirandello e D’Annunzio. Era una sorta di interregno temporale, un momento vuoto in cui gli editori potevano pubblicare i testi senza pagare copyright, e si è assistito a un vero e proprio diluvio di "pirandelli" e "dannunzi" tascabili. Non tutti con risultati apprezzabili e all'altezza di quegli autori, visto che, per non mancare dal mercato, gli editori spingevano per stampare edizioni rapide e con curatele spesso spericolate.

La mappa proposta da Biblioteca Italiana può tuttavia venire integrata senza infrazioni al copyright (in neretto ho segnato i testi aggiunti):

\section{Arrighi-La scapigliatura e il 6 febbraio}

2. Borgese-Rubè

3. Chiari-La filosofessa italiana

4. Collodi-Le avventure di Pinocchio

5. Cuoco-Platone in Italia

6. De Amicis-Cuore

7. D’Annunzio-Il Piacere

8. D'Annunzio-L'Innocente

9. D’Annunzio-Il Trionfo della morte

10. D'Annunzio-Le vergini delle rocce

11. D’Annunzio-Il fuoco

12. D’Annunzio-Giovanni Episcopo

13. D'Annunzio-Forse che sì forse che no

6. In costante aggiornamento bibliografico la banca dati https://www.dirittodautore.it. (si vd. D’Ammassa, Bellantoni e Pellegrino 1999-2019). 
14. D'Azeglio-Ettore Fieramosca

15. De Roberto-Processi verbali

16. De Roberto-Vicerè

17. Fogazzaro-Daniele Cortis

18. Fogazzaro-Piccolo Mondo Antico

19. Fogazzaro-Piccolo Mondo Moderno

20. Fogazzaro-Il santo

21. Fogazzaro-Leila

22. Pisani Dossi-Laltrieri

23. Pisani Dossi-Vita di Alberto Pisani

24. Pisani Dossi-La colonia felice

25. Pisani Dossi-La desinenza in A

26. Foscolo-Le ultime lettere di Jacopo Ortis

27. Manzoni-I Promessi Sposi

28. Nievo-Le Confessioni di un italiano

29. Pirandello-Il fu Mattia Pascal

30. Pirandello-L'esclusa

31. Pirandello-Uno, nessuno, centomila

32. Pirandello-Suo marito

33. Pirandello-Il turno

34. Pirandello-I vecchi e i giovani

35. Palazzeschi-Il codice di Perelà

36. Rovani-Cento anni

37. Svevo-La coscienza di Zeno

38. Svevo-Senilità

39. Svevo-Una vita

40. Tarchetti-Fosca

41. Tommaseo-Fede e Bellezza

42. Tozzi-Con gli occhi chiusi

43. Tozzi-Il podere

44. Tozzi-Tre croci

45. Verga-Eros

46. Verga-Eva

47. Verga-I Malavoglia

48. Verga-Mastro don Gesualdo

49. Verri-Le avventure di Saffo

50. Verri-Le notti romane

Come si può vedere, anche limitando le integrazioni agli autori fuori diritti, le mancanze sono strutturali: mancano due pilastri come D'Annunzio e 
Pirandello, ma anche alcune architravi importanti come Borgese e Palazzeschi, e tutto il secondo Ottocento, più o meno manzonista o scapigliato.

Da questo breve quadro dello stato dell'arte del romanzo digitale emerge lo scollamento tra progetti digitali ed editoriali, e invece, al contrario, l'effetto di condizionamento (probabilmente indiretto) esercitato dal mondo della scuola. Due esempi macroscopici: la riduzione del secondo Ottocento dovuta probabilmente all'effetto-Manzoni, che schiaccia e polverizza la narrativa seguente, e l'isolata presenza di Svevo, dovuta a necessità didattiche, non solo delle scuole superiori, ma dei corsi di Letteratura Italiana Contemporanea, in cui D'Annunzio entra sempre meno, ma non manca mai un corso sveviano. È tuttavia un paradosso, che il romanzo, genere letterario didatticamente più funzionale, manchi di uno strumento didattico. Riflessione che ci conduce direttamente ai casi di studio seguenti: Manzoni, Pirandello e Tozzi.

\section{Tre casi di studio: Manzoni, Pirandello, Tozzi}

Nonostante lo strapotere scolastico, la situazione digitale del romanzo manzoniano non è rosea. Recentemente ho provato a mappare le edizioni on line dei Promessi Sposi, per verificare quali di esse dichiarassero le fonti del testo, e se da queste dichiarazioni fosse possibile ricavare informazioni sull'edizione proposta al lettore, e il risultato è stato poco confortante: delle centinaia di migliaia di edizioni presenti on line, solo quelle presente in Biblioteca Italiana, Letteratura Italiana Einaudi e WikiSource danno indicazioni - e non sempre complete — sul testo di riferimento (Italia 2016). La maggior parte degli studenti legge il romanzo on line (e più spesso i suoi desolanti riassunti), senza saperne la provenienza, la storia testuale, o avere indicazioni sulla sua curatela. La situazione è ora avviata a soluzione grazie al progetto ministeriale italiano PRIN (Progetti di Rilevante Interesse Nazionale) 2017: Manzoni on line, diretto da Giulia Raboni dell'Università di Parma e a cui partecipano anche le Università di Pavia/Cremona, Milano e Bologna e che ha lo scopo di dare un'esistenza digitale alla figura e all'opera di Manzoni, considerata l'assenza in rete di fonti affidabili. Il progetto non è dedicato solo ai testi, che saranno disponibili in versione scientificamente accertata e accompagnati da metadatazione, ma anche ai volumi delle tre biblioteche d'autore: la biblioteca di Casa del Manzoni di via Morone a Milano, quella della Sala Manzoniana di Brera e quella di Brusuglio.? Il

7. Le edizioni manzoniane consultabili in Biblioteca Italiana, codificate con marcatura Xml/TEI negli anni Novanta, saranno aggiornate alle edizioni critiche 
lavoro è iniziato da due anni circa e ha portato già a notevoli risultati, come la mappatura integrale dei segni di lettura: marginalia e postille ai volumi, alcuni del tutto inediti e di straordinario interesse. La marcatura Xml/TEI dei testi permetterà inoltre di individuare, attraverso il riconoscimento delle citazioni, i legami tra i testi e i volumi della biblioteca virtuale così ricostruita, permettendo una navigazione tra gli oggetti digitali del portale. Nel progetto sarà integrata la piattaforma Philoeditor, creata da Fabio Vitali (si vedano Di Iorio et al. 2014; Bonsi et al. 2015), che permette di visualizzare direttamente le variazioni tra Ventisettana e Quarantana, e di mappare alcune categorie individuate dall'utente, ${ }^{8}$ e un prototipo di edizione critica digitale, realizzato sul Conte di Carmagnola, ${ }^{9}$ e sul Fermo e Lucia e gli Sposi Promessi. ${ }^{10}$ L'elemento innovativo è costituito dal fatto che si tratta di un knowledge site e che è il frutto della collaborazione tra varie Università e la Biblioteca Nazionale Braidense, che custodisce gran parte delle carte di Alessandro Manzoni.

Fino al 2017, la situazione di Pirandello non è stata meno grave di quella di Manzoni, perché all'approssimazione dei fake texts, si è aggiunto il forte interesse per i romanzi di Pirandello da parte della scuola, che ha portato alla proliferazione di versioni digitali orfane di curatori, di indicazioni testuali, di introduzione, come la serie proposta dalle edizioni digitali Alphaville, pubblicate nel periodo di "liberalizzazione". ${ }^{11}$ Pirandello è molto letto, rappresentato, recitato, ma a un aumento esponenziale della domanda non ha corrisposto un proporzionale aumento della qualità dell'offerta. A questa situazione pone ora rimedio il portale Pirandellonazionale.it, l'edizione digitale dell'Opera Omnia di Pirandello promossa da Mondadori (l'editore di riferimento del drammaturgo) e realizzata dal CINUM (Centro di Informatica Umanistica) dell'Università di Catania; un progetto ancora in via di definizione, ma di cui mi è possibile anticipare qui alcune linee "guida".12 Si tratta di un portale dedicato all'Edizione Nazionale delle Opere, edizione che, per la prima volta, contempla sia la pubblicazione di volumi dell'edi-

pubblicate negli ultimi due decenni all'interno dell'Edizione Nazionale ed Europea di Alessandro Manzoni, promossa dalla Casa del Manzoni.

8. Sulla piattaforma Philoeditor sta lavorando Ersilia Russo, per una mappatura delle locuzioni idiomatiche e della loro evoluzione dal Fermo e Lucia alla Quarantana (si vd. Russo 2018).

9. Vi lavora, sotto la guida di Francesca Tomasi, Beatrice Nava (cfr. Nava, 2018e).

10. Vi lavora, sotto la guida di Elena Pierazzo, Alessia Martini.

11. Si tratta di edizioni digitali, commercializzate in rete a bassissimo costo, in formato Kindle.

12. Ringrazio Aldo Maria Morace, Antonio Sichera e Antonio Di Silvestro, che mi hanno dato la possibilità di consultare la versione "demo". 
zione critica cartacea che la presentazione dell'edizione digitale, ed è un progetto a forte vocazione didattica. Ogni testo è presentato nei seguenti formati:

1. Riproduzione digitale del manoscritto, realizzata con Pubhtml5, con trascrizione diplomatica a fronte;

2. Edizione digitale sinottica, realizzata con il software Juxta (http://www. juxtasoftware.org/), che mette a confronto le diverse edizioni a stampa: la rivista, la princeps e le successive edizioni in volume;

3. Edizione critica, presentata in un PDF sfogliabile dell'edizione cartacea, che reca a testo la lezione dell'ultima stampa, e presenta in apparato genetico le varianti del manoscritto, della princeps e delle successive stampe;

4. Tavola delle varianti, presentata in un PDF sfogliabile di varianti selezionate e raggruppate in specifiche categorie linguistiche: grafia e fonetica; morfologia e sintassi; lessico; punteggiatura; porzioni di testo modificate;

5. Concordanze del testo, presentate in un PDF sfogliabile con la possibilità di scaricare tavole speciali: forme principali, lemmi principali, forme contrastive.

L'edizione è completata da un elenco dei manoscritti con sedi di conservazione, una tavola sinottica delle edizioni di tutte le opere, la possibilità di accesso ai metadati (di tipo Xml/TEI o RDF), e una sezione dedicata espressamente a strumenti di lavoro pensati per le scuole, come video, riscritture testuali, laboratori linguistici e letterari. A differenza del portale Manzoni on line, questo Pirandello Nazionale è a vocazione più didattica e, grazie alla collaborazione tra Università e Casa editrice, le edizioni, cartacea e digitale, sono realizzate con un'attenzione particolare non solo agli studiosi, ma anche agli studenti e agli insegnanti.

Un modello di tipo diverso, scaturito dalla collaborazione tra Università ed editoria, è in corso di sperimentazione per l'Edizione Nazionale di Federico Tozzi, il cui comitato editoriale ha scelto di collaborare con le Edizioni di Storia e Letteratura nella collana BITeS (Biblioteca Italiana di Testi e Studi), sulla piattaforma Bitesonline.it, per la pubblicazione delle edizioni critiche in formato cartaceo e digitale. La piattaforma permette di leggere il testo sia in formato PDF che in formato digitale, con uno speciale Reader che permette di visualizzare testo, note al piede e note verticali, in spazi virtualmente disponibili per più fasce di apparato o di commento. Il catalogo non contempla ancora romanzi, ma la collaborazione con l'Edizione Nazionale di Tozzi garantirà la prossima entrata di Giovani, a cura di Paola Salatto e 
del Podere, a cura di Tania Bergamelli, mentre è in preparazione, per le cure di Claudia Bonsi e Lorenzo Geri, l'edizione commentata dei Cento anni di Giuseppe Rovani, un altro testo che sarebbe bello potere trovare nella mappa del romanzo che abbiamo presentato.

Le edizioni digitali presentate, che potranno essere analizzate compiutamente quando i progetti saranno terminati, mostrano tuttavia come il romanzo, per lo statuto particolare del genere letterario, per la sua natura polifonica, per le possibilità offerte dagli studi di critica genetica e filologia d'autore che ne hanno messo in luce la genesi stratificata e l'intreccio della sua tradizione manoscritta e a stampa, per le contaminazione con le arti visive e drammatiche, e, non ultimo, per le numerose implicazioni con la didattica, richiedano una infrastruttura digitale più ricca e articolata di quella usata tradizionalmente, una infrastruttura che, senza trascurare la scientificità delle edizioni e la rappresentazione diacronica del testo, venga arricchita dalla contaminazione con riproduzioni documentarie, immagini, video, costituendo attualmente un banco di prova della trasformazione, recentemente auspicata da Gabler $(2018,119)$, dell'edizione critica digitale in un vero e proprio sito di conoscenza:

a digital scholarly edition should, and I hope it will, become a dynamically progressive interactive research site, energised by experiencing a work through its texts, and reciprocally energizing scholarship and criticism, as well as engaged explorative reading, as they search for innovative forms of enquiry and communication.

\section{Oltre: Born Digital Novel}

Con gli anni "Zero" - e con la diffusione presso le giovanissime generazioni delle scritture on line e dei social media - si è assistito a un prevedibile aumento dello sperimentalismo nei generi della scritture dei nativi digitali, passati rapidamente dai blog alle fanfiction (presto trasformate dagli editori in improbabili instant novel), alle scritture sui social network, ma nessuna di queste sperimentazioni è andata al di là del documento sociologico, per evidente incapacità di gestione dei mezzi e degli strumenti della narrazione. La novità del mezzo non ha ispirato alcuna volontà di rinnovamento del genere romanzo, che è proseguito secondo modelli di intrattenimento giovanilistico sentimentale, strenuamente ancorati al più tradizionale dei romanzi di formazione. 
Ne è prova, mero documento sociologico, ma interessante per le dinamiche relative all'uso del mezzo digitale, l'esperimento del sito www. romanzodigitale.it, che al vetusto titolo La ragazza con un fiore tra $i$ capelli, affianca un sottotitolo reso, meno di una decina di anni dopo, già obsoleto: «partitura in sms per telefonini e social network», il cui autore, Davide Romagnoni, e promotore, il consulente di web marketing Marco Prosperi, dichiarano di avere scritto e prodotto per "rendere fruibile un racconto sui principali social media adattando il contenuto, lo stile di scrittura e soprattutto il formato distributivo" (ibidem). L'esilissima trama si svolge esclusivamente tramite sms, distribuiti come post pubblicati giornalmente sul blog del protagonista e sulla propria pagina Facebook e su Twitter. Il romanzo — dichiara la scheda editoriale - "diventa così completamente digitale, un racconto in sms scritto per cellulari e social media: leggendo i post a ritroso è possibile ricostruire tutta la storia e conoscerne lo svolgimento": Diego incontra Arianna una notte di novembre in un locale milanese; da quel momento non ha più pace e decide di conquistare la "ragazza con un fiore tra i capelli". Dalle 8 del mattino alle 21, il lettore segue in diretta le conversazioni di un gruppo di amici impegnati nella faticosa conquista della maggiorata di turno, acchiappata con un Negroni al Plastic, ma che si nega sempre, costringendo il protagonista a una lunga serie di desolanti e reiterate dichiarazioni amorose, noiosissime confessioni all'amico di turno, prevedibilissime e inevitabili bevute. Per ricominciare, alle 8 del mattino seguente, con la giaculatoria degli sms al malcapitato confidente una vera "partitura in sms" (titolo originale, l'unica cosa azzeccata di questa solenne digitazione di tedio): la amo, non so se glielo dico, glielo dico, non glielo dico, chissà se mi ama, forse ama un altro, forse amo la mia ex, ma lei non può più amarmi se non la amo. E così via, per quattro infiniti identici "episodi" (perché "capitolo" è parola vintage, sbandita dal romanzo digitale). Un tedio reso ancora più insopportabile dall'assenza di sintassi, unica malta in grado di tenere insieme, anche in architetture scombinate, le parole che qui invece vagano nel vuoto, abbandonate a loro stesse, e che, in un'impennata di spending review, hanno perso suffissi e vocali: i "cellu" somministrano solo "skerzi", i "raga" stanno "tti bn" e si danno appuntamenti a "dp".

"A voi la scelta" - scriveva il 23 gennaio 2011 su "Libero" Giuliano Tedoldi in una lucida, impietosa recensione - "annoiarvi con la lettura dell'elenco del telefono spacciato da rivoluzione letteraria, o volgervi al rassicurante ma ammuffito neoclassicismo dei nostri cavalieri del romanzo". Questi ultimi, sdegnati e sdegnosi di tanta volgarità, hanno disertato le liquidità digitali, continuando a scrivere trame ottocentesche, raccontate da narratori onniscienti o, nella migliore delle ipotesi, intradiegetici. 
I pochi esempi - come questo - di narrativa digitale sperimentata in rete negli anni Zero restano mummie digitali, in ammuffite pagine web che nessuno aggiorna, pochi navigatori solitari leggono, mentre i "romanzieri digitali" si sono riciclati in promotori della campagna social media delle nuove narrazioni politiche della diretta Facebook, quelle sì popolarissime e seguitissime, orchestrate da narratori onniscienti e amministratori delegati di piattaforme private che gestiscono il consenso — ad vocem - a suon di like, dove la democrazia viene sostituita (copy and paste) da un plebiscitarismo liberticida.

Il romanzo non muore, viva la narrazione contemporanea. Se mezzo secolo fa "libertà era partecipazione", ora "partecipazione è illusione di libertà". Libertà, negli anni Zero, di credere di potere rinnovare il romanzo nel nuovo ambiente digitale, in una realtà piuttosto liquefatta che liquida, senza storie, senza idee e senza una lingua capace di raccontarle. Negli anni Dieci, abbandonati i Blog e le sperimentazioni digitali, si sostituiscono le narrazioni con un'illusione collettiva di esercizio del proprio libero arbitrio, manipolati da abili burattinai, che - veri narratori onniscienti di un plot già scritto - utilizzano la presunta libertà della rete per esercizi di ipertrofismo del proprio ego narcisistico.

Università di Bologna

\section{Opere citate}

Biblioteca Italiana. 1996-2019. Centro Interuniversitario Biblioteca italiana Telematica (CIBIT). @ http://www.bibliotecaitaliana.it/.

Byron, Mark. 2010. "Digital Scholarly Editions of Modernist Texts: Navigating the Text in Samuel Beckett's 'Watt' Manuscripts”. Sydney Studies in English 36: 150-69.

Bonsi, Claudia, Angelo Di Iorio, Paola Italia, Fabio Vitali. 2015. "Manzoni's electronic interpretations". In The Mechanic Reader. Digital Methods for Literary Criticism, special issue of Semicerchio 53.2: 91-99.

Bordalejo, Barbara. 2013. "The Texts We See and the Works We Imagine: the Shift of Focus of Textual Scholarship in the Digital Age". Ecdotica 10: 64-76.

—. 2016. "Los libros del futuro". Humanidades digitales 822 (giugno): 6-8.

2016. "Digital versus Analogue Textual Scholarship or The Revolution is Just in the Title". Digital Philology: A Journal of Medieval Cultures 7.1: 7-28.

Chiari, Pietro. 1753. La filosofessa italiana, o sia le avventure della Marchesa di N.N., scritte in Francese da lei medesima, 3 vol. Venezia: Angelo Pasinelli.

1756. La filosofessa italiana, o sia le avventure della Marchesa di N.N., scritte in Francese da lei medesima, 4 vol. Venezia: Angelo Pasinelli.

D’Ammassa, Giovanni, Raimondo Bellantoni e Raffaella Pellegrino. 1999_ 2019. https://dirittidautore.it. 
Di Iorio, Angelo, Paola Italia, Fabio Vitali. 2015. "Variants and Versioning between Textual Bibliography and Computer Science". In Humanities and Their Methods in the Digital Ecosystem. Proceedings of the Third AIUCD Annual Conference (AIUCD 2014), Francesca Tomasi, Roberto Rosselli Del Turco, e Anna Maria TAмm A Ro, eds. Selected papers, al link https://dl.acm.org/citation.cfm?id=2802614.

Divizia, Paolo. 2012. Dal libro cartaceo a internet e ritorno. Sulla tradizione dei testi nell'epoca del web. Atti del XII Congresso SILFI (Helsinki, 18-20 giugno 2012). Firenze: Cesati: 115-22.

Gabler, Hans. 2018. Text Genetics in Literary Modernism and Other Essays. Cambridge: Open Book Publishers.

Italia, Paola. 2016. "Il lettore Google". Prassi Ecdotiche della Modernità Letteraria 1.1: $1-12$.

Madrignani, Carlo A, ed. 2004. "Introduzione". La filosofessa italiana, o sia le avventure della Marchesa di N.N., scritte in Francese da lei medesima di Pietro Chiari. Lecce: Manni (edizioni originali: Chiari 1753, 1756).

Martinelli, Luciana, ed. 1976. Avventure di Saffo di Alessandro Verri. Ravenna: Longo.

Nava, Beatrice. 2018. Edizione digitale di opere a tradizione complessa: il caso del Conte di Carmagnola, «Patrimoni culturali nell'era digitale. Memorie, culture umanistiche e tecnologia», Book of Abstracts - AIUCD 2018. ISBN 9788894253528. DOI:10.6092/unibo/amsacta/5997, pp. 123-6.

Pierazzo, Elena e Elise Léclerc. 2015. "L'edizione scientifica al tempo dell'editoria digitale". Ecdotica 12: 180-93.

Ross, Shawna. 2016. "Digital Modernism as Method: Recent Publications in Digital Humanities". Journal of Modern Literature 39.3: 163-82.

Russo, Ersilia. 2018. Le locuzioni idiomatiche dal "Fermo e Lucia" ai "Promessi Sposi". Atti del Convegno ADI-Associazione degl'Italianisti, in via di pubblicazione.

Shillingsburg, Peter. 2017. Textuality and Knowledge. State College, Pennsylvania: Penn State University Press.

Zaccarello, Michelangelo. 2017a. "Giornata OPEDIT — Bologna 26 ottobre 2017, cronaca minima”. Prassi Ecdotiche della Modernità Letteraria 3 (https://riviste.unimi. it/index.php/PEML/article/view/9490/8977).

—. 2017b. "Bugiardo come un libro (on line)". Il Sole 24 ore, 4 dicembre (http:// www.ilsole24ore.com/art/cultura/2017-11-27/bugiardo-come-libro-online-130155. shtml?uuid=AEZc4tID\& fromSearch).

. 2018. "Libri senza memoria". Il Sole 24 ore, 21 gennaio (http://www.ilsole24ore. com/art/cultura/2018-01-21/libri-senza-memoria-digitale-081417.shtml?uuid=AEvN4SmD) 